\title{
Multi-channel all-optical signal processing based on parametric effects
}

\author{
Periklis Petropoulos $^{(1)}$, Francesca Parmigiani ${ }^{(1)}$, Kyle R.H. Bottrill ${ }^{(1)}$, Satoshi Yoshima ${ }^{(1,2)}$, Yujia Sun ${ }^{(1)}$, \\ and David J. Richardson ${ }^{(1)}$ \\ (1) Optoelectronics Research Centre, University of Southampton, Southampton, SO17 1BJ, UK. \\ (2) currently at Information Technology R\&D Center, Mitsubishi Electric Corporation, 5-1-1 Ofuna, Kamakura, Kanagawa 247-8501, Japan. \\ E-mail address: pp@orc.soton.ac.uk
}

\begin{abstract}
Two different experiments that use parametric effects for the processing of multiple signals in a single fiber are reviewed. The first experiment uses optical phase conjugation to mitigate the effects of nonlinearity in transmission, whereas the second uses multiple phasesensitive amplifiers to regenerate six different channels.

OCIS codes: (060.2330) Fiber Optics Communications; 060.4370 Nonlinear optics, fibers; (070.5040) Phase conjugation
\end{abstract}

\section{Introduction}

Parametric optical effects based on the third order nonlinearity of optical materials have attracted significant interest in the context of communications in recent years, since they have been shown to be a powerful tool for the processing of fast data signals. Parametric effects present some characteristics that make them particularly suited to the processing of phase and amplitude modulated, broadband signals [1]. They give rise to the generation of an idler at a new wavelength, which emerges from the nonlinear system along with the (amplified) signal, carrying the original modulation of the signal, albeit conjugated. Even though phase conjugation of the idler may appear at first to be an extra complication, it actually turns out to be a useful processing tool, as we will see below. In addition, since nonlinear refraction is virtually instantaneous, parametric effects are generally independent of the data speed.

This automatic scalability to ever-higher data speeds and the resulting benefits in energy efficiency are some of the main attractions of using nonlinear optics to process communication signals. A second important attraction is the potential for processing multiple wavelengths within the same device. Multi-wavelength nonlinear optical processing has been explored for a number of years as a challenging problem to address (see e.g. [2, 3]), impeded mainly by nonlinear crosstalk between the various waves that co-exist simultaneously in the same nonlinear medium. This challenge is easier to address when the power of the signals involved can be kept at low levels, in which case they interact strongly with a much more powerful pump, but not with each other. Therefore, it is possible to satisfy this condition in certain applications of relatively low processing complexity, but not in others, especially when more than one nonlinear processing system needs to be implemented within the same medium.

This paper will review two recent examples of multi-wavelength nonlinear processing, which require different levels of complexity to achieve their goals. The first example uses a single parametric amplifier to simultaneously phase-conjugate six wavelength-division multiplexed (WDM) signals in order to undo the nonlinear impairments that they have suffered during their transmission in an optical link [4]. The second example implements six independent parametric amplifiers in the same highly nonlinear optical fiber (HNLF) to achieve two-level phase quantization (phase regeneration) on an equal number of binary phase-shift keying (BPSK) WDM signals [5]. We will see that whereas the onset of parasitic nonlinear products that could give rise to crosstalk is avoided in the first example of phase conjugation simply by keeping the power of the WDM signals low, the implementation of phase quantization has required careful management of these products, so that they do not compromise the quality of the output.

\section{Nonlinearity mitigation based on optical phase conjugation}

Fiber Kerr nonlinearity represents the primary limitation in the information capacity that can be accommodated in a single channel over long fiber transmission links, since it ultimately restricts the amount of launched power before any nonlinearity-induced distortions and crosstalk damage the signal quality [6]. Significant progress has been achieved in the use of digital back-propagation in recent years, which has allowed fast electronic processing techniques to be used in order to reconstruct the information that has been transmitted, after this has undergone (either linear or nonlinear) deterministic distortions [7]. The complexity of such techniques increases considerably however, when a large number of independent signals co-exist in the same transmission medium, since their collective propagation and nonlinear interactions need to be computed in order to reach the correct solution. An alternative approach to overcoming transmission impairments relies on forcing the signal to repair itself while it is 


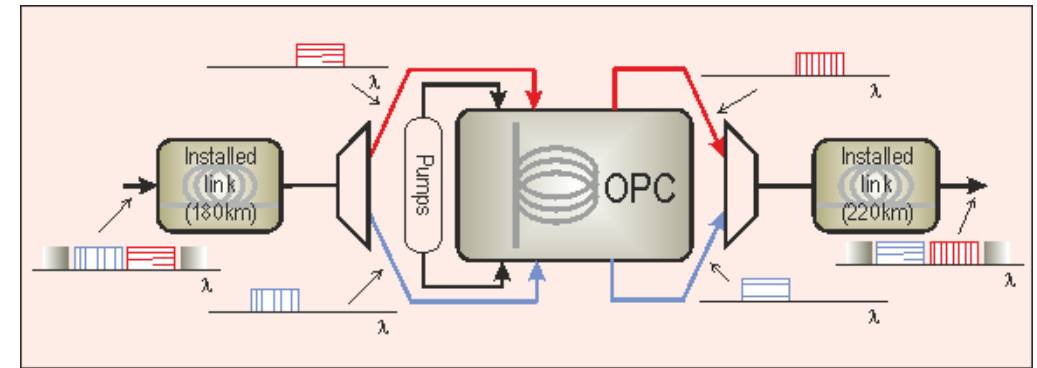

(a)

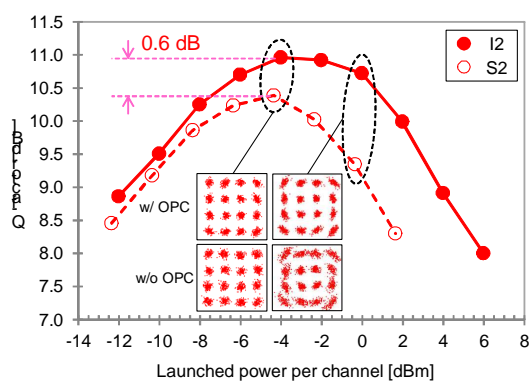

(b)

Fig. 1: (a) Simplified block diagram of the transmission system employing bi-directional optical phase conjugation (OPC); (b) Q-factors vs. launched power for one of the transmitted 16-QAM WDM signals (S2/I2: without/with phase conjugation). For more information, see [4].

being transmitted and is still in an optical form. This can be achieved by adopting a fully symmetric transmission line and placing an optical phase conjugator (as facilitated by a parametric amplifier) at the middle distance. Reversing the signal phase causes any effects that relate to the optical phase (e.g. nonlinear phase modulation, chromatic dispersion) to undo their actions of the first half through propagation in the second half [8]. If transmission is symmetric across its mid-point, then the quality of the transmitted signals will be fully restored at the end of the transmission line. (One should keep in mind that this discussion neglects any stochastic noise introduced e.g. by amplifiers along the transmission line.)

Phase conjugation in an optical parametric amplifier can be effected through the generation of an idler wave, which in the general (non-degenerate with respect to the signal) case, results in the generation of new frequencies. Consequently, phase reversal of the signal usually requires the reservation of an unoccupied spectral band, which clearly limits the practicality of the scheme. In the experiment we review here, we split the spectral band containing the signals to be conjugated into two halves and phase-conjugated each half in the place of the second (see Fig.1a) [4]. This was achieved in a cost-efficient way, using the two opposite directions of the same HNLF to give rise to two independent parametric amplifiers that shared the same pump sources $[4,9]$. Each of the phase conjugating parametric amplifiers was a two-pump non-degenerate system, while aligning the pumps in orthogonal polarizations ensured polarization-insensitive operation.

The system was tested in an installed fiber transmission link with a total length of $400 \mathrm{~km}$ (part of the UK's Aurora2 National Dark Fibre Infrastructure Service). The phase conjugator was placed close to the middle of the transmission line, within the limits imposed by the physical topology of the installed infrastructure. This implied that the first part of the link was shorter than the second; therefore, the link was not completely symmetric. Testing the system in such non-favorable conditions is relevant, since it is considered that topological restrictions in the placement of the phase conjugator will always be present in practical systems. Thirteen WDM signals were transmitted in total, nine of which were modulated with 16-QAM signals and four with OOK, all at 10 Gbaud. Six of the 10 Gbaud 16-QAM signals were applied to the phase conjugator, divided into two bands of three channels each, as described above. Figure $1 b$ outlines the results obtained for one of the channels at the end of the transmission line and compares the system performance with and without use of the phase conjugator. The figure shows that use of the phase conjugator allowed for a wider range of launched powers to be used for optimum performance. Overall, $\sim 0.6 \mathrm{~dB}$ improvement in the measured Q-factor was observed. The constellation maps with and without phase conjugation for two different launched powers are also reported in Fig. $1 \mathrm{~b}$. The reduction in phase noise through the use of phase conjugation is visible for all symbols, but is especially noticeable for the outer symbols of the constellation. More recently, we reinforced our conclusions by transmitting 64-QAM signals.

\section{Phase-quantization in a polarization-assisted phase-sensitive amplifier}

Signal regeneration requires the implementation of step-like transfer functions, which are challenging to implement using (analogue) optical processes. Phase-sensitive amplifiers (PSAs) have proven to be extremely effective in realizing steep phase transfer functions, and thus regenerating phase-shift keying signals [10]. Phase regeneration based on phase-sensitive amplification is implemented by interfering a signal with its (coherent) idler generated through the process. Therefore, the spectral arrangements for the pumps and signals are quite specific, and the regeneration of multiple signals generally requires the co-existence of a number of independent parametric amplifiers. In such a setting, the management of spurious pump-to-pump and pump-to-signal nonlinear interactions presents significant challenges.

To address these challenges and achieve the regeneration of six WDM BPSK channels in a single fiber, a number of actions were taken in the experiment we outline here [5]. Firstly, the multiple pump pairs required for the 


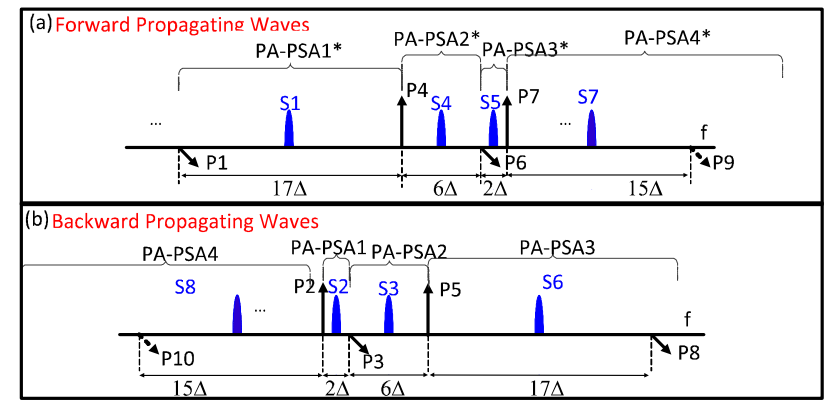

(i)

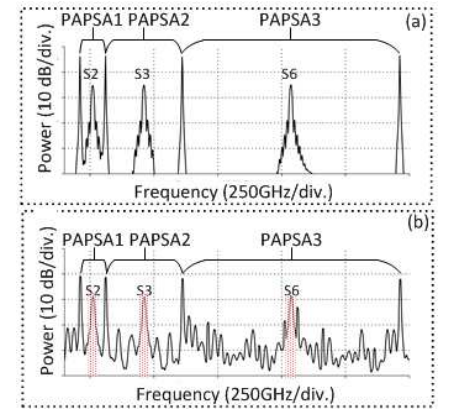

(ii)
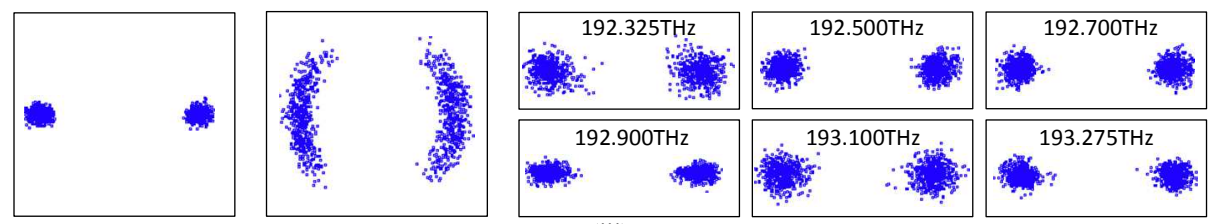

(iii)

Fig. 2: (i) Pump and signal spectral allocations in the two directions of the HNLF in the 6-channel phase quantization system; (ii) example of measured spectral traces (a) before and (b) after the HNLF for one direction of the fiber; (iii) Signal constellation diagrams: back-to-back (left), after introduction of noise (middle), at the system output (right). For more information, see [5].

various PSAs were orthogonally polarized relative to each other. This drastically reduced the pump-to-pump nonlinear interactions by $\sim 30 \mathrm{~dB}$. It also allowed the use of even very low parametric gains for the coherent interaction between the signals and their degenerate idlers in order to give rise to phase regeneration, through a scheme that we call polarization-assisted phase-sensitive amplification [11]. In addition - as in the previous experiment - the HNLF used for the implementation of the PSAs was employed in a bi-directional fashion, with three channels being processed in each direction. The placement of the pumps and signals in each fiber direction was engineered in such a way that any parasitic nonlinear components would be placed outside the signal wavelengths (see Fig.2a-b). Using this configuration, it was observed that the strongest pump-to-pump spurious FWM components were $\sim 5 \mathrm{~dB}$ weaker than the output signals, whereas the remaining pump-to-signal spurious components were more than $10 \mathrm{~dB}$ weaker than the signals. However, in all cases, no parasitic components overlapped spectrally with the regenerated signals. Both constellation diagrams (see Fig.2c) and bit-error rate measurements confirmed regeneration with an optical signal-to-noise ratio improvement between 0.55 and $1.2 \mathrm{~dB}$ for all channels.

\section{Conclusions}

Through the presentation of two different experiments, this paper discussed the challenges present in the nonlinear processing of multiple wavelength channels in optical parametric amplifiers. Depending on the application, multiple channels may be processed through either a single or multiple individual parametric amplifiers that co-exist in the same nonlinear medium. Whereas power engineering can be effectively employed in the former case, significant challenges relating to the management of spurious nonlinear effects need to be addressed in the latter case, which will effectively restrict the number of channels that can be usefully processed in a single system.

\section{Acknowledgement}

This work was part of the EPSRC Photonics Hyperhighway Programme Grant. The optical conjugation experiments were performed on the jointly EPSRC/JISC-supported NDFIS Aurora2.

\section{References}

[1] M.E. Marhic, "Fiber Optical Parametric Amplifiers, Oscillators and Related Devices", Cambridge University Press, 2007.

[2] F. Parmigiani et al., "Progress in Multichannel All-Optical Regeneration Based on Fiber Technology," JSTQE, 18, 689-700 (2012).

[3] M. Vasilyev and T.I. Lakoba, "All-optical multichannel 2R regeneration in a fiber-based device," Opt. Lett. 30, 1458-1460 (2005)

[4] S. Yoshima, Y. Sun, K. R. H. Bottrill, F. Parmigiani, P. Petropoulos, and D. J. Richardson, "Nonlinearity mitigation through optical phase conjugation in a deployed fibre link with full bandwidth utilization," in ECOC'2015, paper We.2.6.3.

[5] F. Parmigiani et al., "Multi-channel Phase Regenerator Based on Polarization Assisted Phase-Sensitive Amplification", PTL (2015) to appear.

[6] R. J. Essiambre et al., "Capacity trends and limits of optical communication networks," Proc. IEEE, 100, 1035-1055 (2012).

[7] E. Ip, "Nonlinear compensation using backpropagation for polarization-multiplexed transmission," JLT, 28, 939-951 (2010).

[8] K. Solis-Trapala et al., "Nearly-Ideal Optical Phase Conjugation based Nonlinear Compensation System," in OFC'2014, paper W3F.8.

[9] V. J. F. Rancano et al., “100-GHz grid-aligned multi-channel polarization insensitive black-box wavelength converter," JLT, 32, 3027 (2014).

[10] R.Slavík et al.,"All-optical phase and amplitude regenerator for next-generation telecommunications systems,” Nature Phot., 4, 690 (2010).

[11] F. Parmigiani, G. Hesketh, R. Slavik, P. Horak, P. Petropoulos, D.J. Richardson, "Polarization-Assisted Phase-Sensitive Processor", JLT, 33(6), 1166-1174 (2015). 